\title{
Protein microarray-based IgE immunoassay for allergy diagnosis
}

\begin{abstract}
Protein microarray is a miniaturized multi-analyte, solid-phased immunoassay where thousands of immobilized individual protein spots on a microscopic slide bind are bound to specific antibodies (immunoglobulins) from serum samples, which are then detected by fluorescent labeling. The image processing and pattern recognition are then quantitatively analyzed using advanced algorithms. Here, we describe the use of an in-house-produced complex protein microarray containing extracts and pure proteins that has been probed with antibodies present in the horse sera and detection by fluorophore-conjugated antibody and data analysis. The flexibility of the number and types of proteins that can be printed on the microarray allows different set of specific IgE immunoassay analysis to be carried out.
\end{abstract}

Keyword: Protein microarray; Component-resolved diagnostics; Microarray-based allergy testing; Horse sera; IgE-binding assay; Partial Least Squares Discriminant Analysis (PLSDA); Variable influence on the projection (VIP) 